\title{
Entrelacs
}

ENTRELACS Cinéma et audiovisuel

11 | 2014

La Voix

\section{La correspondance filmée de José Luis Guerin et Jonas Mekas : des voix en écoute}

Filmed correspondence between José Luis Guerin and Jonas Mekas: listening voices

Cécile Tourneur

\section{OpenEdition}

\section{Journals}

Édition électronique

URL : http://journals.openedition.org/entrelacs/1486

DOI : 10.4000/entrelacs.1486

ISSN : 2261-5482

Éditeur

Éditions Téraèdre

Référence électronique

Cécile Tourneur, «La correspondance filmée de José Luis Guerin et Jonas Mekas : des voix en écoute », Entrelacs [En ligne], 11 | 2014, mis en ligne le 07 janvier 2015, consulté le 01 mai 2019. URL http://journals.openedition.org/entrelacs/1486 ; DOI : 10.4000/entrelacs.1486

Ce document a été généré automatiquement le 1 mai 2019.

Tous droits réservés 


\title{
La correspondance filmée de José Luis Guerin et Jonas Mekas : des voix en écoute
}

Filmed correspondence between José Luis Guerin and Jonas Mekas: listening voices

\author{
Cécile Tourneur
}

1 La correspondance filmée qui s'établit entre les cinéastes José Luis Guerin et Jonas Mekas s'inscrit dans le cadre d'un projet intitulé "Toutes les lettres, correspondances cinématographiques ». Il trouve son origine dans les premiers échanges de ce type entre Victor Erice et Abbas Kiarostami qui se sont déroulés entre 2005 et 2007. À leur suite, Jordi Balló, le directeur artistique du Centre de culture contemporaine de Barcelone de 1999 à 2011, propose à cinq cinéastes hispanophones de correspondre à leur tour avec un cinéaste de leur choix ${ }^{1}$. Parmi eux, José Luis Guerin désigne Jonas Mekas comme l'un de ceux avec lequel il souhaiterait mener cette expérience. Deux rendez-vous sont nécessaires pour officialiser le début de leurs échanges et se mettre d'accord sur le procédé : «Ils filmeront quatre lettres chacun, et Jonas propose que ce soit José Luis qui commence l'échange $\rrbracket^{2}$. Le résultat, finalement constitué de neuf lettres entre novembre 2009 et avril 2011 - Guerin débute et clôt la correspondance - est destiné à être présenté sous la forme d'une installation, mettant en écho les films dans un espace muséal. «Exposer les lettres filmées ${ }^{3}$ procède de la volonté des commanditaires de «créer un espace public [...] une ruelle de cinéma où les visiteurs se rencontrent, parlent, se regardent ou échangent leurs sensations $[. ..] »^{4}$. La circulation réelle et matérielle des lettres durant le projet trouve son équivalent dans le dispositif de projection choisi, un "tiers-espace $"^{5}$ se dessine alors entre les deux cinéastes et le public avec qui ils partagent ces objets cinématographiques. Cet espace est également le lieu de résonnance des voix des cinéastes qui vont être émises, se faire entendre, mais aussi devenir l'écho de l'autre et des autres, résultat d'une écoute profonde. 
2 À travers les démarches épistolaires et autobiographiques de Mekas et Guerin, le rapport que le cinéaste entretient avec sa propre voix est à interroger, alors qu'il est lui-même preneur et "fabricant" de son. Les éléments vocaux présents dès la première lettre orientent profondément les modalités d'intervention de la voix dans la suite de la correspondance. Ce phénomène est le résultat d'une écoute partagée, que Mekas et Guerin mettent chacun leur tour en scène. Enfin la voix apparaît comme une des possibilités d'adresser ses images à l'autre, et devient une des clés de voûte de l'échange épistolaire. La voix est comprise comme étant «le support acoustique de la parole " selon la définition de Guy Cornut, et sera explorée en premier lieu en tant que manifestation sonore à travers son lien au corps et à l'espace cinématographique ainsi crée.

\section{La lettre filmée, une pratique artisanale}

José Luis Guerin commence à filmer dans les années 1970, alors que l'Espagne est en prise avec le régime franquiste. Son œuvre, constituée aujourd'hui d'une dizaine de films, n'est pas définie par un seul genre mais traversée par la fiction, le documentaire, l'essai. Principalement montrée en salle, elle s'enrichit aussi d'une installation vidéo en 2010-2011, «La Dama de Corinto ». La lettre filmée s'intègre parfaitement dans l'œuvre qu'il a construite méticuleusement, révélant à la fois un cinéma intime et généreux, profondément personnel mais tourné vers l'autre ${ }^{7}$. Jonas Mekas, cinéaste américain d'origine lituanienne, initiateur du journal filmé au milieu des années 1960, est aujourd'hui l'auteur d'une œuvre colossale et multiple qui se poursuit aussi longtemps que sa vie. En 1969, Diaries, Notes and Sketches (Also known as Walden)" journal $»^{9}$, marque le début d'une orientation de sa pratique cinématographique qui lie son parcours d'homme et de cinéaste. Il est également reconnu pour ses activités d'éditeur et rédacteur de la revue Film Culture à partir de 1955, critique au Village Voice à partir de 1958 et membre fondateur du New American Cinema Group en 1960. La FilmMakers' Cooperative en 1962, suivie de la Film-Makers' Cinematheque en 1964, puis de l'Anthology Film Archives en 1970, ouvrent de nouveaux espaces de création, de diffusion et de parole autour des films aux États-Unis. Familier de la pratique épistolaire au cinéma, il a tout d'abord adressé ses films à d'autres, notamment à ses enfants Oona et Sebastian ( Paradise Not Yet Lost, aka Oona's Third Year ${ }^{10}$ en 1979 et The Education of Sebastian or Egypt Regained $^{11}$ en 1992), puis à partir de la fin des années 1990, certains de ses films sont explicitement intitulés « lettres » : Letters to Friends...from Nowhere...Video Letter \#1 ${ }^{12}$ (1997), Letters from Nowhere / Laiškai iš Niekur N.1. ${ }^{13}$ (1997) et A Letter from Greenpoint (2003) ${ }^{14}$. À partir du milieu des années 2000 , la lettre tient une place importante dans sa pratique cinématographique, comme le rapporte le critique Jon Davies : «Mekas a déclaré que la lettre - une communication intime entre deux individus - était désormais devenue son mode favori à la fois en vidéo et poésie $»^{15}$. Cependant, l'échange mis en place avec Guérin est encore inédit pour le cinéaste, puisque ses lettres précédentes n'attendaient pas de réponses et ne se construisaient pas en fonction de ces dernières.

4 Josep Ramoneda - directeur du CCCB de 1998 à 2011 - décrit le projet global de la correspondance filmée proposée aux cinéastes comme « un retour à l'artisanat » :

« [...] des cinéastes qui ont bien voulu revenir à la dimension la plus élémentaire et essentielle du cinéma: une caméra à la main, des situations, des images, un montage et quelqu'un de l'autre côté qui attend des nouvelles ». 
Ces caractéristiques sont déjà constitutives de la pratique cinématographique de Mekas à travers le journal filmé, qui s'est inscrit dans une tradition du film artisanal, parfois qualifié d'amateur, dont le sens a été redéfini et revalorisé notamment par Maya Deren ${ }^{16}$ et Stan Brakhage ${ }^{17}$. Ce dernier, employant l'expression home movie, (souvent traduite trop rapidement par «film de famille») parle d'une pratique cinématographique qui se déroule « à la maison, seul, sur des films qui n'avaient [n'ont] apparemment pas de valeur commerciale $»^{18}$. Le cinéma de Guerin trouve aussi sa source dans une certaine forme d'artisanat dont il explique les enjeux ainsi :

«Aussi, quand dans les années 1970, j’ai commencé à filmer en super-8, le cinéma libre et poétique de Jean Cocteau et Jonas Mekas nous mettait à l'abri de l'amateurisme et de l'apprentissage, qui pouvait être une conséquence mais pas une motivation. Maintenant que l'amateurisme a disparu, j'éprouve une grande estime pour ce mot à racine latine qui procède de l'amour et suppose un artisanat $»^{19}$.

Les films composant l'échange épistolaire entre Guerin et Mekas pourraient être qualifiés de home movies. Home, le foyer au quotidien, parfois quitté, puis retrouvé, est aussi l'espace intime de création et de pensée des films, notamment à travers la présence dans les lettres filmées des lieux mêmes où elles se fabriquent (le banc de montage $16 \mathrm{~mm}$ de Mekas apparait dans sa deuxième lettre, en réponse, Guerin filme ses outils numériques). Home, renvoyant à un point d'ancrage, cœur de sa propre existence et de celle des films, devient l'espace, de part et d'autre, au sein duquel prend vie l'échange épistolaire. C'est également le foyer qui accueille les voix de ces cinéastes, au sein d'un dispositif cinématographique qui les place en position de preneurs d'images et de sons.

\section{Des cinéastes à l'image et au son}

$7 \mathrm{Au}$ cours des lettres filmées qu'il envoie à Jonas Mekas, José Luis Guerin déploie un paysage cinéphile sans frontière spatiale ni temporelle, évoquant aussi bien Stanley Donen, Robert Flaherty, Georges Meliès, Friedrich Wilhelm Murnau, Fritz Lang, Yasujiro Ozu que le destinataire de ses lettres. Mais dans sa première lettre, Guerin se réclame d'une lignée cinématographique remontant aux débuts du cinématographe: celle des opérateurs Lumière, employant ce nouveau medium pour appréhender leur environnement immédiat. Descendants de ces hommes parcourant le monde avec leur caméra, Guerin et Mekas conservent des fragments visuels et sonores de ce qui les entoure, selon le credo énoncé par Mekas lors de leur première rencontre et repris par Guerin dans sa lettre : «I react to life »" ${ }^{20}$. Chez Mekas comme chez Guerin, cette " réaction à la vie » se fait par l'intermédiaire de leurs corps, appareillés d'une caméra, enregistrant à la fois l'image et le son. La caméra est d'ailleurs perçue par Mekas, depuis le début de sa pratique en $16 \mathrm{~mm}$, comme un prolongement de son corps ${ }^{21}$. Même si ses outils ont depuis changé, il a conservé cette approche fusionnelle avec l'utilisation des caméras vidéo ${ }^{22}$. Les corps de ces cinéastes, ne faisant qu'un avec la caméra, sont aussi les lieux d'émission de leurs voix, qu'elles soient synchrones (le cinéaste parle en filmant, hors champ ou face caméra) ou enregistrées après le tournage, lors des opérations de montage. Lorsque le corps du cinéaste n'est pas présent dans le champ, la voix filmée est « un seuil entre visible et invisible. Elle se réfère au corps qui la porte, et en même temps le représente dans son absence, donnant à ce corps absent de l'image quand même une "image", nous le faisant imaginer, le désignant, le dessinant en creux $»^{23}$. 
8 La voix, même hors champ, asynchrone, est l'extension indéniable d'un corps, traversé par le souffle rendant seul possible l'émission de sons articulés. Le corps de Guerin, que nous ne voyons jamais directement dans le champ, est prolongé par sa voix ${ }^{24}$. La voix de Mekas parcourt la majorité de ses films argentiques, mais l'enregistrement synchrone du son direct n'est jamais exploité. Son utilisation de la vidéo à partir des années 1980 introduit en revanche une synchronisation, y compris labiale, très fréquente. Mekas se filme en gros plan, face à la caméra, et parle. Guerin souligne le fait que l'introduction de sa propre voix dans ses lettres a été guidée par le fait que son correspondant cinématographique soit justement Mekas :

«Cela s'est passé avec difficulté parce que j'avais énormément de pudeur à mettre ma voix. Ce fut une difficulté énorme mais, en même temps, je trouvais que c'était le seul choix naturel de faire la lettre, avec la voix, en pensant à Jonas. En faisant la correspondance avec un autre cinéaste que Jonas, peut-être que j'aurais préféré introduire un texte dans le film $»^{25}$.

Indifféremment des dispositifs choisis pour s'enregistrer et se faire entendre, la prise en considération par Guerin de l'importance de la voix de Mekas dans son œuvre antérieure détermine dès la première lettre la place qui lui sera accordée.

\section{“Écrire” une lettre au cinéma}

Les éléments qui définissent les films échangés comme étant des lettres sont doubles, à la fois oraux et écrits. L'écrit permet souvent de signifier, par l'usage d'intertitres, le début et la fin de la lettre. Le système varie peu: Guerin utilise une typographie blanche 
générée informatiquement sur fond noir, tandis que Mekas préfère filmer son écriture manuelle.

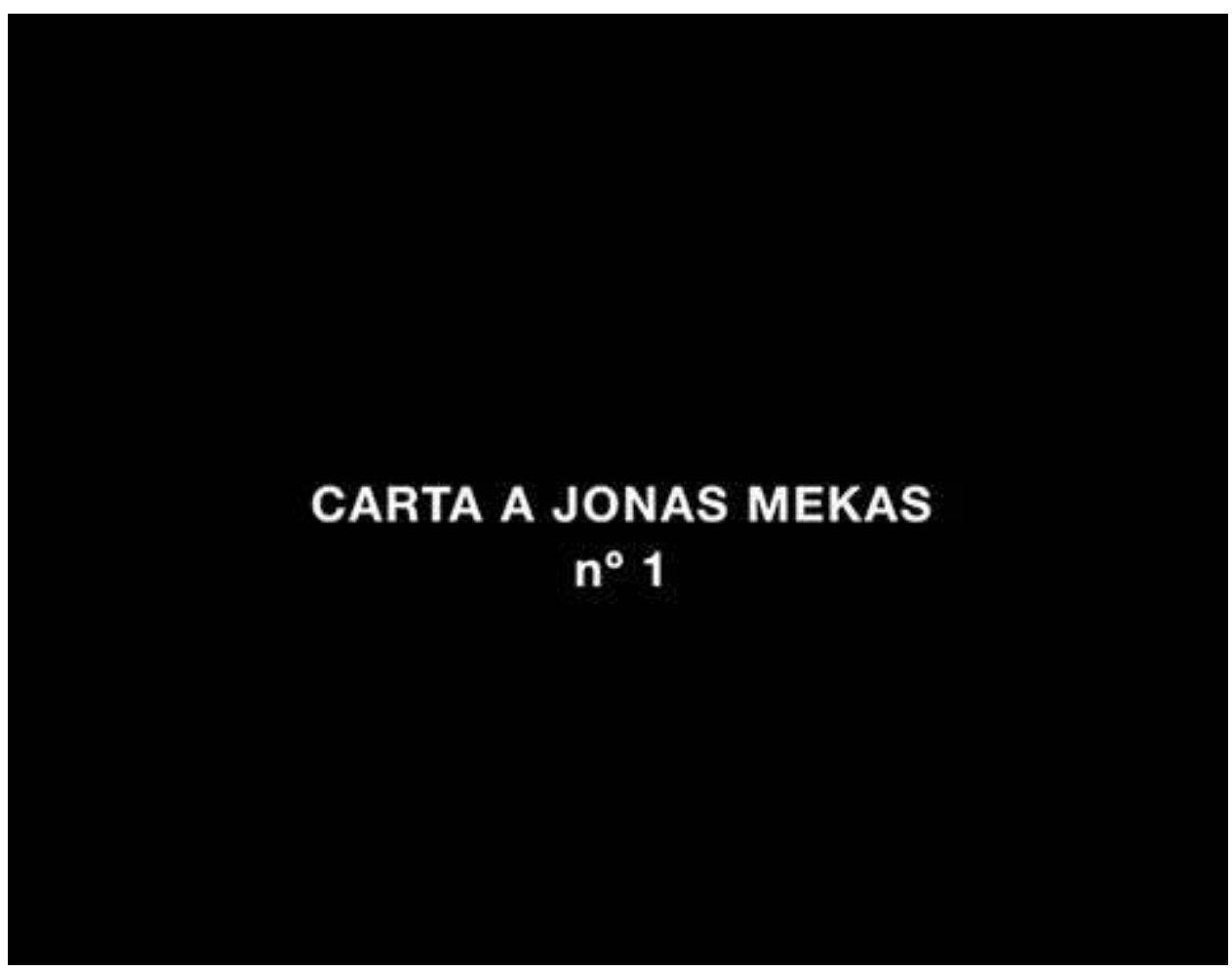

Carta a Jonas Mekas $n^{\circ} 1$

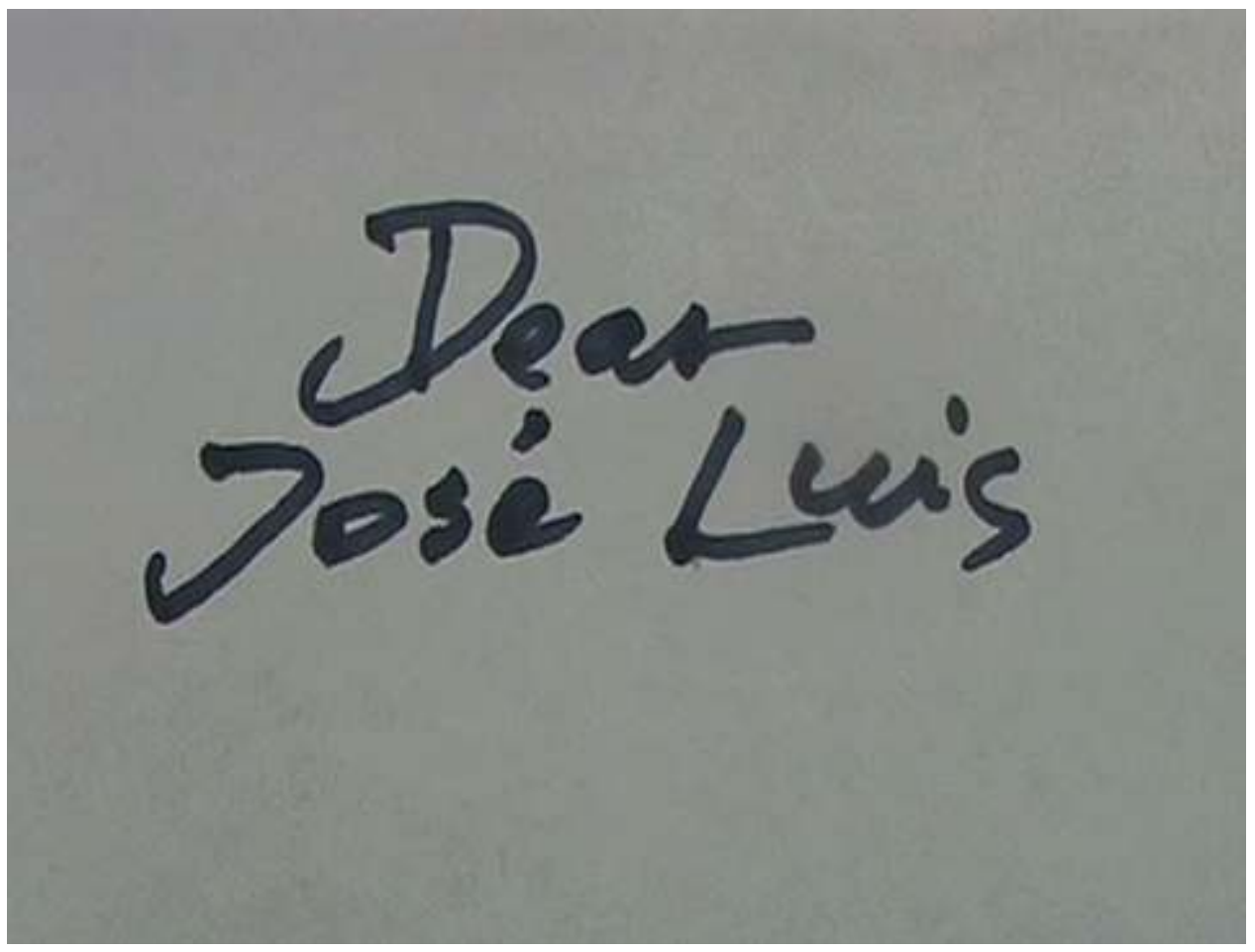

Dear José Luis

11 Le recours à la voix dans ce même but - identifier le film comme étant une lettre - a en revanche un impact beaucoup plus important sur la construction des lettres suivantes apportées en réponse. La voix se déploie et évolue en fonction des interventions vocales 
précédentes. Alain Bergala identifie l'importance du premier geste cinématographique vers l'autre :

«Celui qui envoie la première lettre, dans un échange épistolaire, a un grand pouvoir, celui de donner le ton. Dans une correspondance vidéo, où les choix initiaux sont très ouverts, le premier coup est décisif, souvent, pour la couleur future de la correspondance $»^{26}$.

Les termes employés par Bergala à ce propos sont singuliers : le ton et la couleur peuvent renvoyer au champ lexical de la peinture, mais aussi de la musique, en particulier aux composantes sonores de la voix qui se singularise par ces deux éléments. Les propositions vocales faîtes par Guerin, et notamment le ton employé dans sa première lettre, orientent la façon dont Mekas va répondre et en tenir compte.

José Luis Guerin transpose à l'oral les conventions épistolaires apparaissant traditionnellement à l'écrit. Il emploie le mode d'adresse classique d'un échange par lettre, en commençant par ces mots très simples, énonçant le lieu, la date ( « Paris, ocho de noviembre 2009 ») et le destinataire, accompagné d'une formule amicale («Querido Jonas » ${ }^{27}$ ). Nous remarquons qu'il s'exprime en catalan, dans sa langue maternelle, supposant un sous-titrage ou une traduction accompagnant la lettre à l'attention de Mekas. La lettre débute à son arrivée à Paris. Elle est alors réalisée à l'occasion d'un voyage, Guerin ne retranscrit pas un quotidien familier, mais l'expérience d'un éloignement de son foyer. $\mathrm{Au}$ contraire, la première lettre envoyée par Jonas Mekas se déroule chez lui à New York, at home. Il accuse réception de la lettre filmée de Guerin, en reprenant les mêmes conventions à l'oral, scellant, par la voix, l'échange et engageant une symétrie dans la construction du film qu'il adresse à son tour : « Dear José Luis, thank you for your letter, now here is my little letter $\aleph^{28}$. Un mimétisme s'installe entre les cinéastes, la tonalité est affectueuse, bienveillante, Jonas Mekas baptiste même Guerin dans sa troisième lettre " My friend in cinema $»^{29}$; nous avons l'impression de rentrer dans l'intimité de leur correspondance. La durée des lettres est également à peu près équivalente, un système d'échange se met en place et chacun le respecte ${ }^{30}$. De même que dans la lettre de Guerin, la voix de Mekas est d'abord entendue sans que le corps du cinéaste n'apparaisse dans le champ de la caméra. Cependant, comme le souligne Bergala à ce propos, « quand Mekas prend la parole dans ses lettres, même lorsqu'il est absent de l'image, c'est son corps qui est présent dans le plan, incarné dans son débit déclamatoire, sa voix éraillée, au timbre unique $»^{31}$. La signification de mots prononcés en préambule n'a finalement que peu d'importance, ceux-ci étant assez conventionnels, mais une musicalité se met en place d'une lettre à l'autre, par la répétition des motifs vocaux, légèrement différents selon les langues employées - l'américain ou le catalan - leurs sonorités et leurs aspérités. Nous pourrions parler de "variations $»^{32}$, en reprenant le champ lexical de la musique. À l'exception de la deuxième lettre, où nous entendons d'abord la voix de pêcheurs sur le lac de Walden où Guerin se rend ${ }^{33}$, ses autres lettres débutent invariablement par " Querido Jonas $»^{34}$. Mekas prend quant à lui plus de liberté, s'adressant directement à José Luis Guerin sans ces conventions, notamment dans ses deuxième et troisième lettres.

14 Au début de sa première lettre, Guerin filme son carnet ouvert. Il a griffonné puis raturé quelques mots sur les pages blanches, devenus illisibles. Seul le mot «film» est resté intact, tandis que l'espace sonore se remplit de sa voix et de mots qui décrivent le contexte de la "fabrication" de la lettre. 


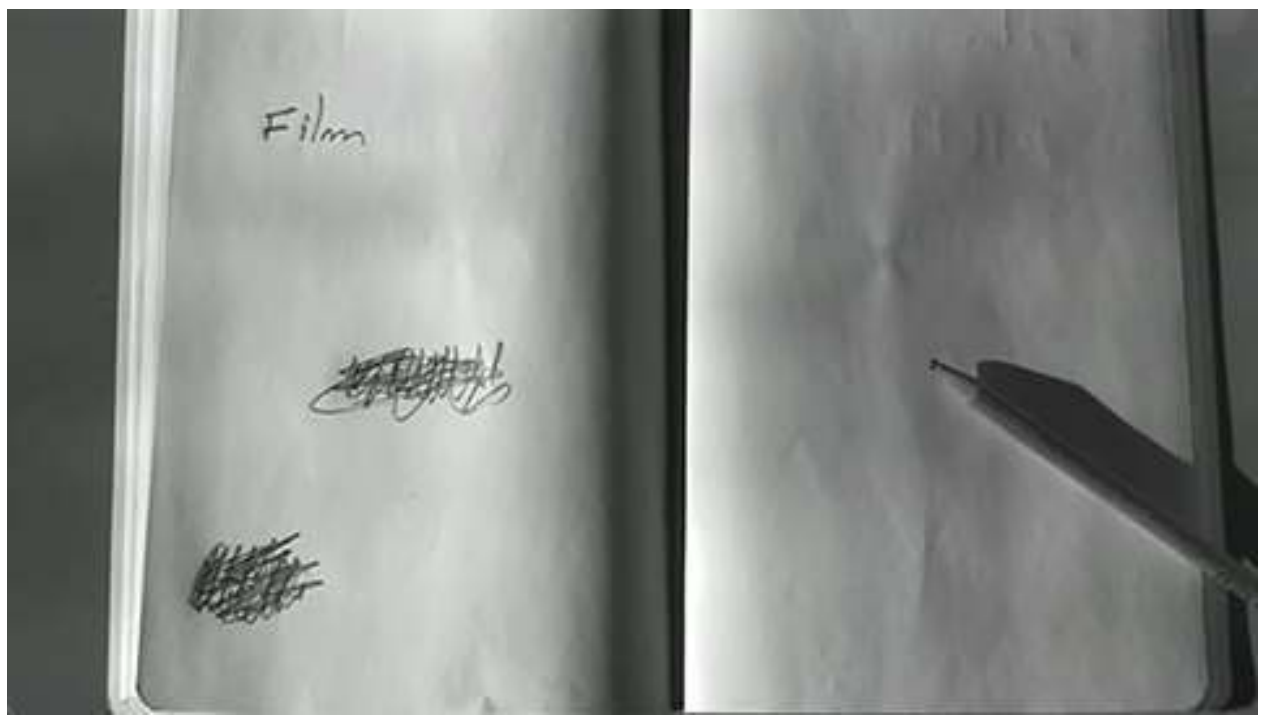

Carnet de Guerin

La tentative d' "écrire" une lettre de façon classique semble être un échec, comme en témoignent les ratures. Le film apparait donc comme la solution permettant d'appréhender la réalité complexe de ce voyage, dans toutes ses strates, aussi bien visuelles que sonores. Dès lors, la voix de Guerin se peuple de sonorités étrangères, de légers décrochages dans les modulations de la voix faisant pénétrer les noms étrangers des lieux qu'il traverse à Paris : "Gare de Lyon », "Centre Pompidou ». L'appropriation d'un lieu autre, lors d'un voyage, passe souvent par la capacité à prononcer correctement les noms des rues, des places, des monuments, mais l'accent persistant peut aussi signifier une forme de retenue à se fondre dans cet espace qui n'est pas le notre :

"L'accent est aussi le résultat, le reste, d'un duel entre deux langues sur le territoire de l'une ou de l'autre, c'est-à-dire la trace d'une résistance qu'a opposée l'une à une forme d'acculturation par l'autre. Autrement dit, s'il reste encore quelque chose d'une langue première dans la pratique d'une langue seconde, l'accent est ce reste, cette dernière velléité de résister $»^{35}$.

Guerin joue avec ces sonorités étrangères qui traversent sa propre langue, ce «horschamp qui reste ouvert dans les coulisses de la parole $»^{36}$, alors qu'il explicite la place qu'il s'est choisie dans le monde pendant deux années, celle d'un voyageur perpétuel acceptant les sollicitations venant de festivals du monde entier et qu'il nomme en anglais - la langue comprise par le plus grand nombre - «guest $»^{37}$.

Dans les lettres qui vont suivre, qu'elles soient réalisées par Mekas ou Guerin, les sonorités véhiculées par la co-présence de différentes langues, et induites par la première lettre, vont continuer à se mêler. Jonas Mekas, résidant aux États-Unis depuis 1949, a luimême toujours conservé les traces audibles de sa langue maternelle, apportant un rythme singulier, une rugosité qui rappelle ses origines et son enfance passée dans la campagne lituanienne. La cartographie vocale et sonore qui se dessine au sein de ces échanges épistolaires est complexe, déterminée par les allers-retours des deux cinéastes entre ce que Mekas appelle (dans sa troisième lettre) le Nouveau et l'Ancien Monde, et les personnes rencontrées, d'horizons divers, dont les voix composent également la bande sonore. Dans sa première lettre, nous entendons Mekas discuter avec une jeune femme tous deux restent hors champ - et nous percevons qu'elle n'est pas américaine, mais probablement française. Mekas confirme notre intuition en employant, en français, le 
mot « réalité » pour tenter de lui expliquer son rapport à la vie (ce que lui considère comme étant « réel » et ce qu'elle nomme, selon lui à tort, une "attitude»). Dans sa quatrième lettre, Mekas filme le cinéaste Dominique Dubosc expliquant en anglais - avec un fort accent français - la situation problématique entre le Brésil et le Paraguay: le premier envahit un peu plus chaque année le territoire du second, en dépassant la frontière d'une centaine de mètres chaque jour. Cet exemple devient alors métaphorique des frottements langagiers et vocaux que l'on entend à travers les lettres, les intrusions d'une langue dominante au dépit d'autres plus régionales, mais dont les sonorités et donc l'identité qui y sont associées persistent.

\section{Des cinéastes à l'écoute}

La répétition des motifs vocaux d'une lettre à l'autre - les conventions épistolaires transposées à l'oral comme l'introduction de sonorités étrangères qui semblent se propager - provient d'une écoute réciproque entre les cinéastes, comme le souligne Jordi Balló :

«Il s'agit d'une correspondance, c'est-à-dire qu'il existe un échange, qu'une lettre regarde et écoute la précédente, et que c'est ainsi que la relation épistolaire se construit ${ }^{38}$.

Mais, reprenant les interrogations soulevées par Jean-Luc Nancy au sujet de l'écoute, nous nous demandons :

"Qu'est-ce donc être à l'écoute, comme on dit "être au monde"? Qu'est-ce qu'exister selon l'écoute, pour elle et par elle, qu'est-ce qui s'y met en jeu de l'expérience et de la vérité ? Qu'est-ce qui s'y joue, qu'est-ce qui y résonne, quel est le ton de l'écoute ou son timbre, l'écoute serait-elle elle-même sonore ? " ${ }^{39}$

Notre corps, qui résonne lorsque notre voix se fait entendre, recueille aussi les sons lorsque nous écoutons, les reçoit comme autant de vibrations qui le conduiront à agir en conséquence. Nous pouvons en observer un exemple à travers la façon dont une musicalité vocale et instrumentale se fait entendre dans la deuxième lettre de Guerin, suite à l' "écoute" de la lettre envoyée par Mekas. À la fin de celle-ci, Mekas est filmé sur le quai du métro jouant de la trompette - instrument impliquant l'intervention du souffle pour la formation de sons musicaux, comme la voix implique le souffle pour la formation de sons vocaux. Guerin, après avoir pris connaissance de cette lettre, explique son envie de recueillir à son tour les sons musicaux de la ville dans laquelle il séjourne : «Le jour suivant, entendant encore l'écho d'une trompette résonnant dans la métro, je vais en ville, à Boston, pour écouter un peu de musique ». Il filme alors sur le quai du métro une femme en train de chanter a cappella, puis dans la rue un homme portant un transistor à l'épaule, faisant résonner dans l'espace urbain, les sonorités d'une musique sudaméricaine - qui rappelle par ailleurs un mambo écouté chez Mekas sur lequel il filme Benn Northover, son fils Sebastian, quelques amis et lui-même en train de danser. Dans la construction du montage, les sons sont en avance de quelques secondes sur les images, anticipant ce que l'on va voir, jouant avec l'imaginaire des spectateurs quant à leur provenance. Dans le premier cas, il s'agit d'une voix enregistrée "en direct", Guerin recueille le son de la voix auprès de la personne même qui l'émet. Le second enregistrement sonore témoigne en revanche d'une médiatisation des sons musicaux entendus par le biais du transistor, Guerin n'a pas directement accès aux personnes les produisant. La bande sonore de la lettre qui se constitue, issue de prélèvements sonores 
dans le réel, participe à la composition d'une géographie vocale et sonore mouvante, déplaçant les frontières.

Des rencontres que Guerin filme au cours de ses lettres ne sont restituées le plus souvent que les moments d'écoute de sa part, pendant lesquels il recueille, pour le destinataire de ses lettres, l'image et la voix de son interlocuteur. L'enregistrement de la voix d'autrui, en même temps qu'il filme, se fait le plus souvent dans une grande proximité : les plans sont serrés, les regards vers la caméra fréquents, appuyés. Guerin semble vouloir percer le mystère de cette émission vocale, de son invisibilité, en allant au plus près, avec sa caméra, de son lieu d'émission, c'est-à-dire le corps, le visage. Même hors champ, silencieux, Guerin est omniprésent et son attention palpable. Dans cette même lettre réalisée à Boston, Guerin filme un ami compositeur qui évoque sa cinéphilie. Nous entendons cette fois encore la diversité et la confrontation des langues, notamment par la prononciation de titres de films (la Maman et la putain de Jean Eustache). Guerin ne répond pas aux questions de son ami - qui lui demande quel est son film préféré de Fred Astaire mais il le laisse parler, suivre le fil de sa pensée ${ }^{40}$. Ces questions restant ouvertes, elles peuvent aussi bien s'adresser à Mekas qu'aux spectateurs qui prend connaissance de la lettre. Dans sa troisième lettre, à l'occasion d'un festival où il est invité à Lisbonne, Guerin recueille auprès des personnes présentes des témoignages sur les différentes cinématographies mondiales : au Canada, en Allemagne, en Slovénie. Le lieu de tournage de cette séquence, un bateau, se prête particulièrement bien à ces pérégrinations orales à travers les différents territoires cinématographiques, symbolisant un entre-deux, redessiné par ces personnes qui viennent témoigner des paysages artistiques, culturels, de leurs pays respectifs. Tous s'expriment en anglais, avec des accents prononcés, renvoyant également, par les aspérités modulant leurs voix, à un ailleurs. Guerin s'attarde particulièrement sur une jeune femme slovène, Nika Bohnic, qui réalise des interviews des cinéastes et avec laquelle il parvient à inverser les rôles d'interviewer et d'interviewé. Guerin, censé parler de lui, filme et écoute pourtant à deux reprises Nika évoquer le cinéma de son pays et sa situation problématique, mais aussi des moments plus intimes de sa vie : son enfance, ses premières expériences de spectatrice. L'essentiel de ce portrait réside dans le regard et la voix de Nika, qui apparaissent comme un miroir tendu au cinéaste, ce vers quoi il aspire (l'acuité du regard, la justesse de la voix). Guerin nous informe du destin tragique de Nika dès le début de sa lettre. Les images permettent de conserver une trace visuelle des personnes disparues. Le cinéma, par l'illusion du mouvement qui le constitue, présente un simulacre de la vie même. L'enregistrement de la voix a aussi cette faculté d'actualiser le souvenir, mais au contraire du défilement des images que nous pouvons figer, il n'est pas possible de l'arrêter : le son, aussitôt écouté, s'évanouit. Insaisissable et éphémère, il se déroule toujours dans le présent de l'écoute, alors qu'il est plus aisé de retenir dans une image, même gelée, l'écho d'un mouvement.

Mekas met également en scène son écoute, à l'occasion d'un voyage dans l'Ancien Monde, en Pologne et en Slovaquie. S'il ne répond pas directement à l'émotion de Guerin à propos de Nika, nous notons malgré tout des points de convergence par sa façon de filmer à son tour une jeune femme - sa fille Oona - qui devient la porte-parole de l'histoire douloureuse des lieux traversés. Contrairement à Nika, Oona ne témoigne pas de sa propre vie. Le rôle qu'elle endosse est celui d'un guide : ses interventions orales ont pour fonction de donner des informations - ponctuées de commentaires plus personnels - à ses compagnons de voyage, lors des visites du cimetière profané à Cracovie et des lieux de détention et de torture à Banská Štiavnica. Les voix de ces jeunes femmes sont alors 
porteuses d'histoires qui les dépassent, mais qui les constituent aussi d'une certaine façon: Nika par l'histoire chaotique de son pays, Oona, liée à l'Europe par les racines lituaniennes de son père ${ }^{41}$.

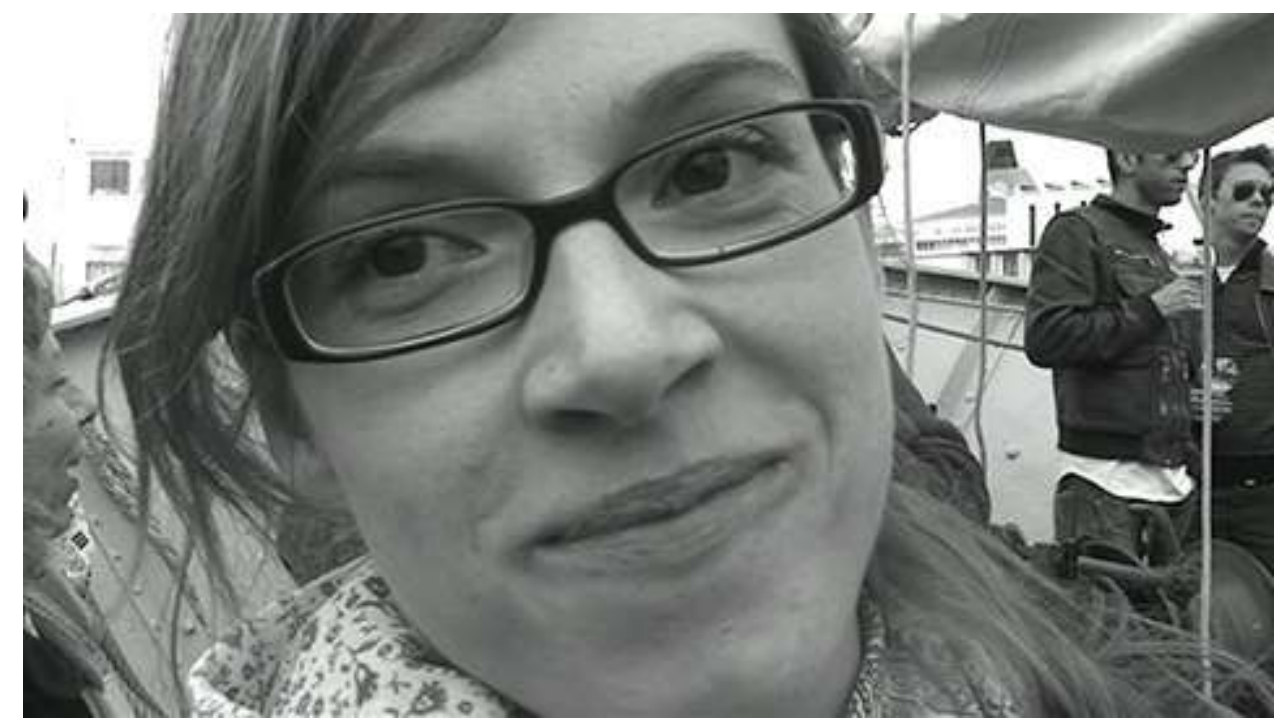

NIKA BOHNIC

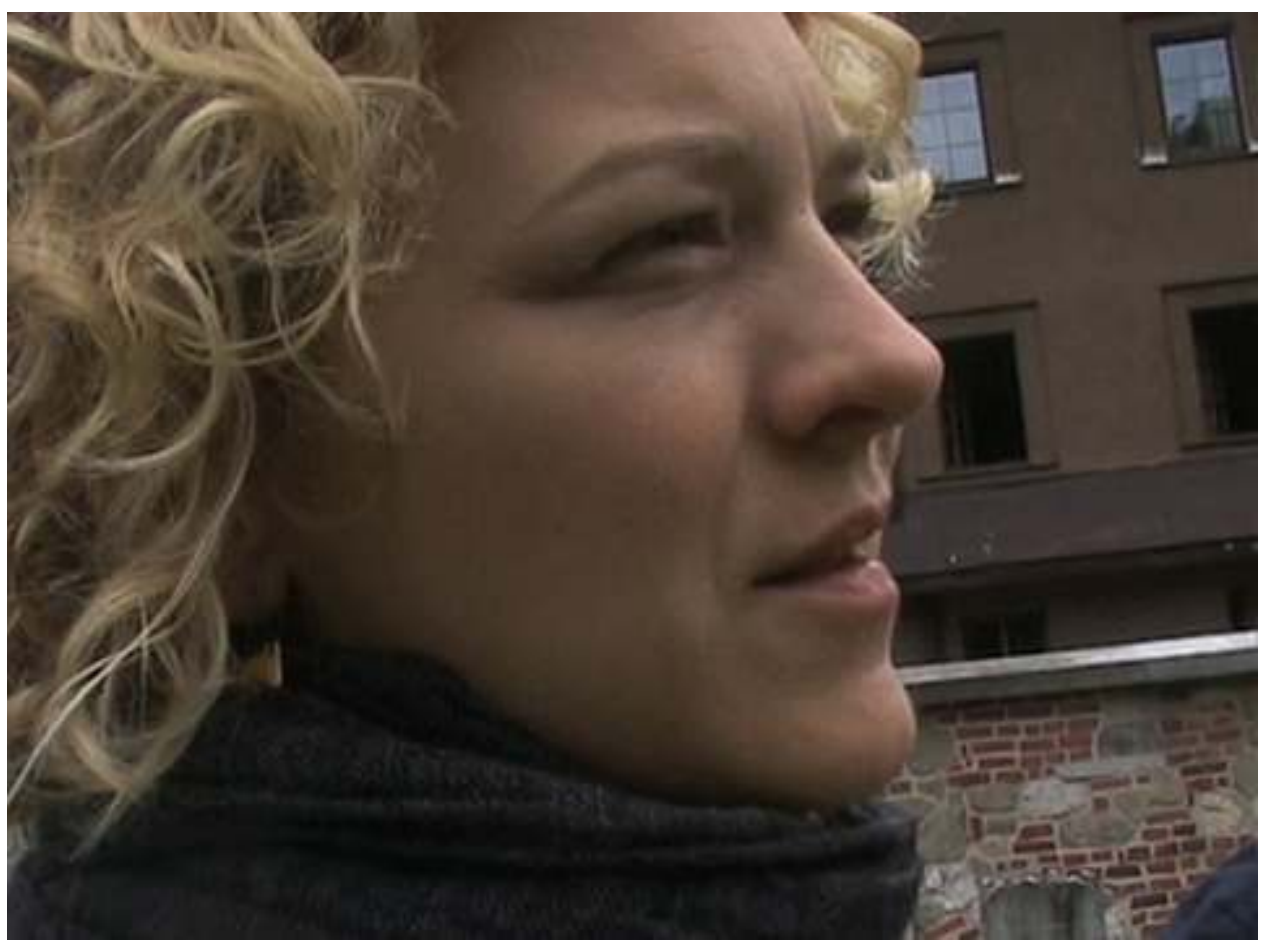

OONA MEKAS

Cette symétrie entre le recueil des voix des deux jeunes femmes révèle à la fois, de la part des cinéastes, une capacité d'écoute envers les personnes filmées, mais aussi une écoute entre eux, créant au fil des lettres des correspondances sonores et visuelles. Mekas signifie ainsi à Guerin, sans évoquer directement Nika, qu'il a entendu et pris acte de ce que celui qu'il nomme «my friend in cinema » a souhaité lui transmettre. Au cours de la séquence à Banská Štiavnica, Mekas filme son ombre sur le mur d'un des cachots, empruntant alors la signature de Guerin dans ses lettres (même si Mekas, dans de nombreux films, est aussi coutumier de cette mise en scène). Cette ombre est à la fois la 
preuve qu'il est bien là, mais peut également être interprétée comme une façon, entre autres, de certifier à l'autre que ces images et ces sons lui sont bien destinés. La silhouette qui se découpe rappelle la présence du corps du cinéaste derrière la caméra, mais dans une posture humble, se positionnant en retrait face à ce qu'il filme.

\section{Adresser les images et les sons} où il était invité. Nous n'avons pas, comme chez Mekas, la présence du corps du cinéaste dans le champ ou juste derrière la caméra, commentant "en direct" les images. Même si cette intervention vocale s'apparente davantage à une voix off, celle-ci n'exclut pas forcément la présence du cinéaste :

"L'enregistrement d'une voix off n'engage pas simplement la nécessité de donner un texte et de l'énoncer correctement; la place, la distance, l'adresse sont des variables décisives et non pas secondaires. La neutralité des voix proches éteint les enjeux, d'où la nécessité d'entendre dire tout un corps mouvant, rassemblé dans la parole, une parole donnée dans un espace signifiant $»^{45}$.

Sa voix, résonnant dans l'espace cinématographique ainsi construit, lui permet de signer et d'adresser ses images à l'autre. La lettre s'ouvre sur le plan d'un écran blanc de cinéma monté sur une place en Pologne. Guerin a filmé, devant cet espace vierge, les allées et 
venues des passants. Il relève à l'oral les moments de tension, de calme dans la chorégraphie des corps devant l'écran et traversant le champ. Les modulations de sa voix, ses intonations, les pauses et les silences, l'introduction de sonorités étrangères, semblent être les équivalents sonores des mouvements des $\operatorname{corps}^{46}$. Puis, à Venise, Guerin définit sa position de cinéaste dans le monde, travaillant principalement dans l'espace public. Il filme des personnes qui occupent et font vivre ce territoire, alors qu'elles n'en sont pas originaires, et qu'il nomme " personnes déplacées $»^{47}$. Il identifie plusieurs d'entre elles et les fait surgir, par la voix, de la foule : « le roumain et son accordéon, le vendeur de roses pakistanais, l'africain et ses sacs ». Chacun est isolé quelques secondes et retrouve une identité, une dignité, à la fois par le cadre et par la voix. À la fin de cette lettre, nous entendons une voix féminine, s'exprimant dans une langue que nous ne comprenons pas. Guerin présente alors à Jonas son amie Malgorzata Zavinska, jeune femme polonaise vivant à Venise - une "femme errante » comme Guerin la décrit - faisant écho à la façon dont Mekas introduit ses amis dans ses films.

La voix, avec des dispositifs différents, ne se contente pas seulement d'orienter la perception des images, mais donne du relief à ce qui est présent dans leur silence. À la fin de son exploration des images du passé dans sa deuxième lettre, Mekas s'arrête sur un gros plan de son visage filmé trente ans auparavant, nommant, en riant «the filmmaker himself $»^{48}$, se retrouvant ainsi face à lui-même. Guerin semble répondre à ce face-à-face entre le cinéaste et son image en filmant son propre reflet dans la pupille de son amie, plan qu'il agrandit à l'extrême. Ce plan, fixe, contient l'essence du cinéma de Guerin et 
par extension de Mekas: le cinéaste n'existe que dans ce que la personne filmée lui renvoie de son propre regard et de sa propre écoute.

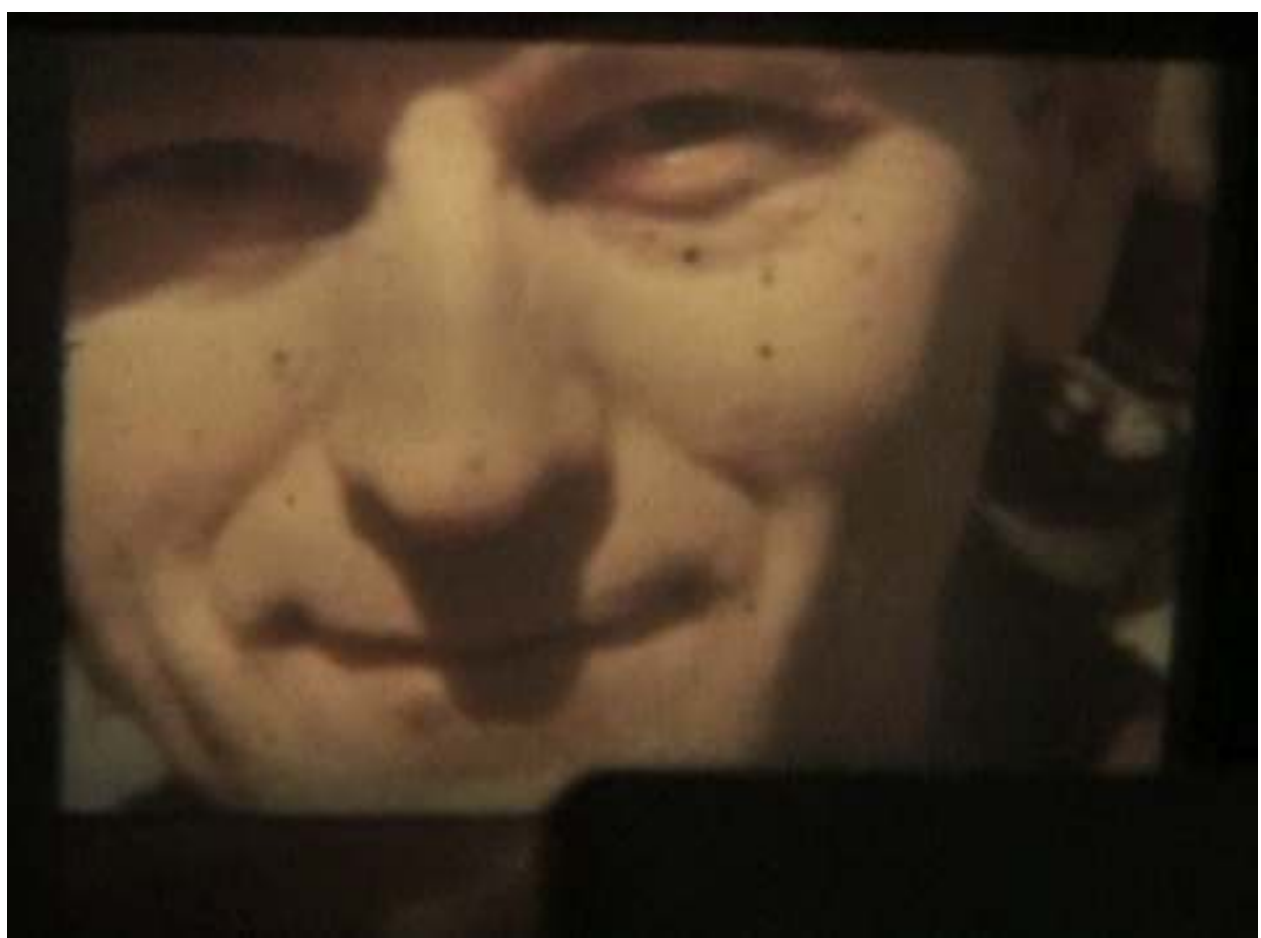

Portrait de Mekas

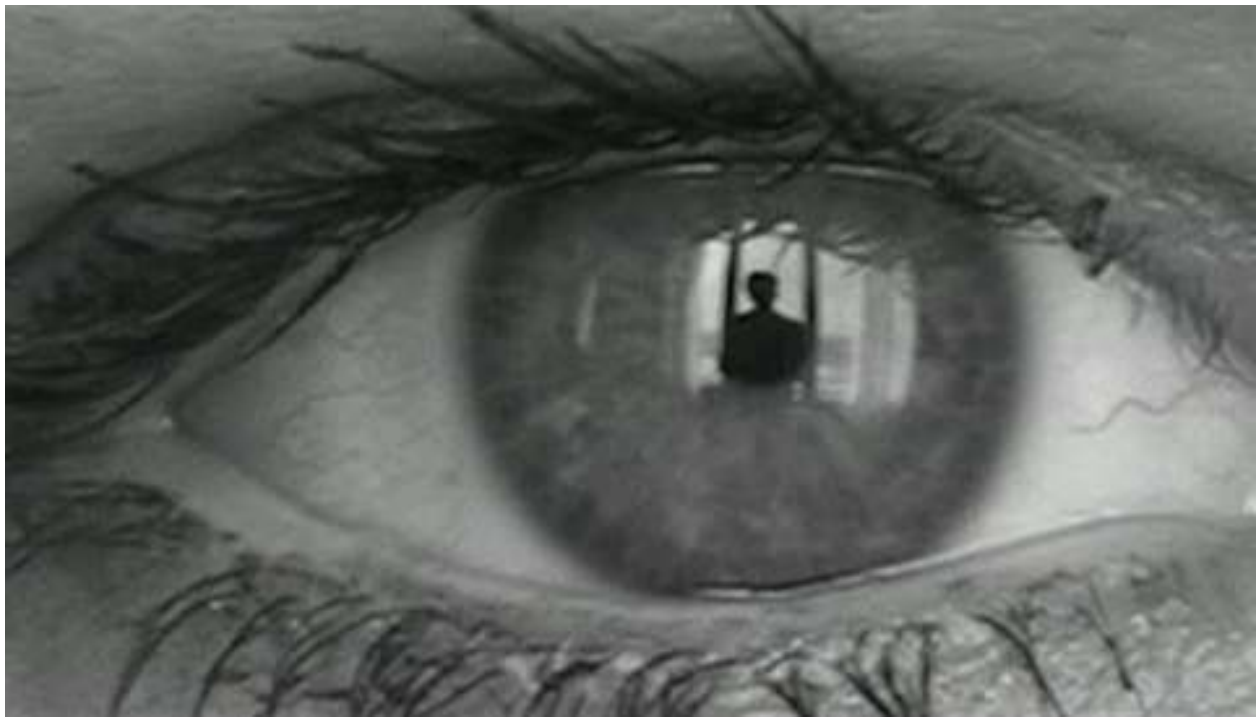

Portrait de Guerin

\section{Vers une extinction de la voix...et la fin de la correspondance}

Guerin envisage sa dernière lettre comme une "glose » orale sur Mekas, s'effectuant selon un mode "familier ", " presque intime » ${ }^{49}$, qui renvoie à ce foyer de fabrication des films, home. Il met en scène quelques objets évoquant le cinéma underground des années 1960 aux États-Unis et ses échos arrivés jusqu'à lui, jeune cinéaste dans les années 1970. Il 
filme une édition espagnole du Movie Journal de Mekas, des bobines $16 \mathrm{~mm}$, le premier album Velvet Underground tournant silencieusement sur une platine 33 tours.

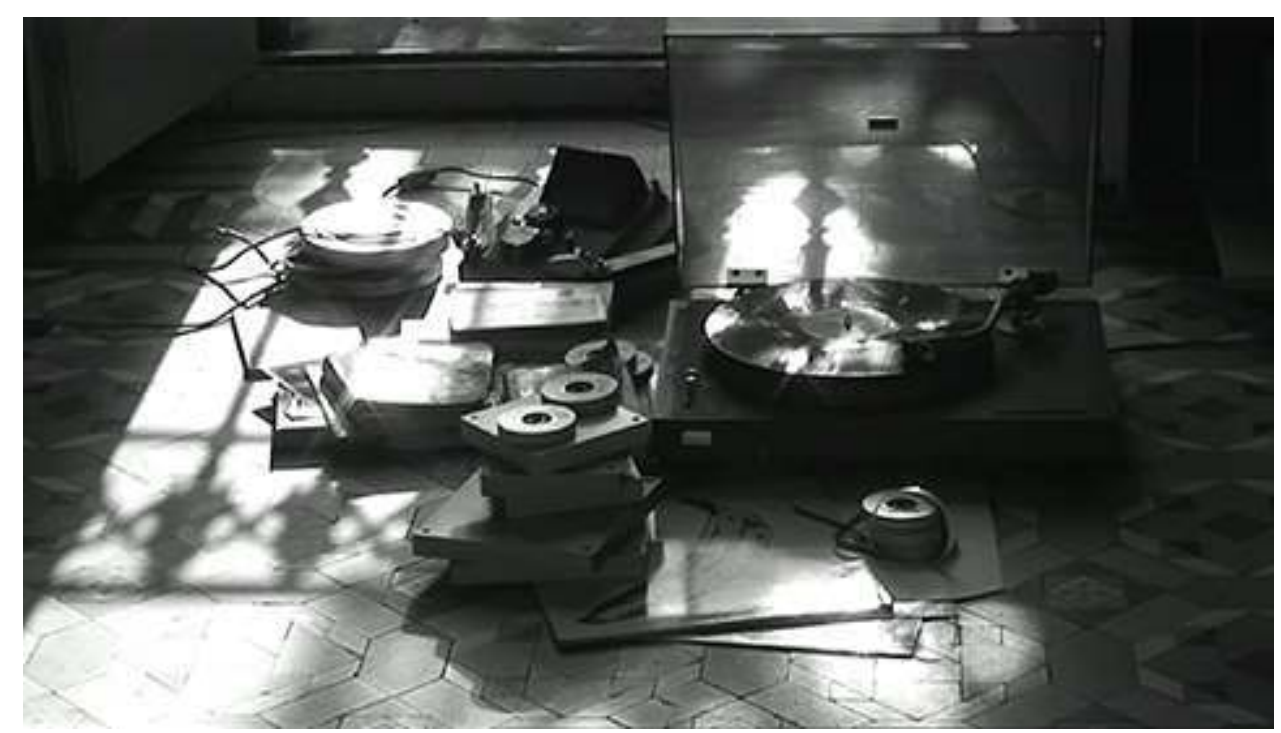

Hommage à Mekas une chorégraphie qui semble parfaitement millimétrée, tandis que Guerin évoque les différentes communautés d'artistes travaillant ensemble dans l'histoire du cinéma; passant de la Factory d'Andy Warhol à celle de Mack Sennett, de la Keystone à la «fabrique » de Kouléchov, du nouveau cinéma brésilien à la Shochiku. Guerin, à la fois à travers ses images et le commentaire qu'il en fait, porte une attention particulière au travail en collectivité et sa disparition dans le cinéma actuel. Il considère Mekas comme étant l'un des derniers représentants de ce cinéma fabriqué, vécu, pensé au sein d'une communauté. Nous retrouvons ici un procédé assez similaire à l'enregistrement des passants devant l'écran blanc en Pologne: les modulations de la voix donnent une pulsation au va et vient des travailleurs qui traversent le champ. Mais le projet d'hommage à son correspondant se trouve modifié par l'accident nucléaire de Fukushima qui survient en mars 2011, et Guerin décide alors d'envoyer à Mekas des images qu'il a tournées un an auparavant au Japon, notamment lors de sa visite sur la tombe d'Ozu.

À partir de la moitié de la lettre, la voix de Guerin se tait, et plus aucune voix ne sera entendue: les rires des femmes nettoyant les tombes ${ }^{50}$ et jouant avec l'objectif de la caméra ne résonneront pas au-delà du silence des images. Le bruit du vent est comme un souffle persistant, tandis que Guerin s'attache au laborieux travail de deux fourmis, filmées en très gros plan, tentant de transporter une brindille. La lettre se clôt sur un plan de la pierre tombale d'Ozu, sur laquelle est gravé l'idéogramme japonais « $M u$ », traduit littéralement par "vide", principe lié au bouddhisme zen dans lequel le vide, l'absence, sont perçus comme une valeur positive. Le vide revêt autant d'importance et de signification que les éléments qu'il entoure ${ }^{51}$. Bien que nous ne puissions pas entendre de voix, il est possible de percevoir en fond sonore le bruit du vent, de la pluie, des éléments naturels du lieu filmé. Cette inaudibilité vocale permet finalement de mettre en relief et de faire résonner tout ce qui a circulé à l'oral durant la correspondance. L'extinction de la voix signifie la fin des échanges, mais son écho continue de tisser un lien entre les cinéastes. 
voix semble donc être le squelette des lettres au cinéma, ce qui maintient la communication par-delà des déplacements géographiques et du temps qui sépare chaque envoi. Si Guerin, le premier, tend à employer des conventions épistolaires pour communiquer avec Mekas, nous nous apercevons que ce qui se joue de part et d'autre dans les interventions orales s'enrichit de tonalités, de sonorités qui renvoient perpétuellement à un imaginaire et à un ailleurs, encore accentués par les nombreux voyages. La notion d'échange dans la correspondance, menée de façon extrêmement assidue et studieuse par les deux cinéastes, donne au film une troisième dimension, un «tiers-espace » comme nous l'avons baptisé en introduction, renvoyant à ce home, cœur de cette pratique autobiographique. Cet espace est aussi le lieu privilégié de l'émission vocale, livrant une parole bien sûr, mais surtout la présence de corps, ceux de cinéastes en état d'attention permanente, de disposition totale face au monde, en écoute.

\section{BIBLIOGRAPHIE}

BALLÓ Jordi, « Les lettres filmées », dans Jordi Balló (dir.), Todas las cartas : correspondencias filmicas, catalogue de l'exposition (Mexico du 27 avril au 31 juillet 2011, Barcelone du 12 octobre 2011 au 19 février 2012), Barcelone, Éditions Intermedio, avec la coopération du Centre de culture contemporaine de Barcelone, Acción Cultural Española, le Centre culturel universitaire Tlatelolco, la Casa Encendida de Madrid, 2011, pp. 329-332.

BERGALA Alain, «Je t'écris ces images...», dans Jordi Balló (dir.), Todas las cartas : correspondencias filmicas, catalogue de l'exposition (Mexico du 27 avril au 31 juillet 2011, Barcelone du 12 octobre 2011 au 19 février 2012), Barcelone, Éditions Intermedio, avec la coopération du Centre de culture contemporaine de Barcelone, Acción Cultural Española, le Centre culturel universitaire Tlatelolco, la Casa Encendida de Madrid, 2011, pp. 332-340.

BRAKHAGE Stan, « Défense de l'amateur », trad. de l'américain par Pierre Camus, dans Yann Beauvais, Jean-Michel Bouhours (dir.), Le Je filmé, catalogue de l'exposition (Paris, Musée national d'art moderne, 31 mai au 2 juillet 1995), Paris, Éditions du Centre Pompidou, 1995, pp. 1969-1960 [1 $1^{\text {ère }}$ éd. « In Defense of amateur », dans Filmmakers Newsletters 4 (9-10), été 1971].

CAVALIER Alain, entretien avec Jean-Pierre Limosin et Serge Toubiana, « Les pulsations de mon cœur, le 29 avril 1996 », Les Cahiers du Cinéma, n504, juillet/août 1996, pp. 42-49.

COMOLLI Jean-Louis, «L'oral et l'oracle, séparation du corps et de la voix », Images Documentaires, $\mathrm{n}^{\circ} 55 / 56,1^{\mathrm{er}}$ trimestre 2006, Paris, pp. 13-38.

CORNUT Guy, La Voix, Paris, Presses Universitaires de France, 2009 [1 ${ }^{\text {ère }}$ éd. 1983].

DAVIES Jon, « Brief Glimpses of Beauty: Jonas Mekas at the 2005 Venice Biennale », Cinemascope, $\mathrm{n}$ '25, [En ligne] 2005 [Consulté le 31/03/2014]. Disponible sur : http:// www.mayastendhalgallery.com/jonas_cinema_scope/index.html.

DEREN Maya, « Amateur versus professional », dans Essential Deren, collected writings on film by Maya Deren, Kingston, New York, Documentext, 2005, pp. 17-18 [1 $1^{\text {ère }}$ éd. Movie Makers Annual, 1959]. 
DESHAYS Daniel, Entendre le cinéma, Paris, Éditions Klincksieck, 2010.

DIEUTRE Vincent, « Abécédaire pour un tiers-cinéma ». La lettre du cinéma, n²1, 2003, pp. 75-85.

FLEISCHER Alain, L'Accent : une langue fantôme, Paris, Éditions du Seuil, 2005.

GUERIN José Luis, septembre 2012, trad. de l'espagnol par André Gabastou. Texte introductif à la brochure accompagnant sa rétrospective au Centre Pompidou, à Paris, du 30 novembre 2012 au 7 janvier 2013, dans le cadre de la manifestation « José Luis Guerin / Jonas Mekas, cinéastes en correspondance ", pp. 2-3.

GUERIN José Luis, propos recueillis par Renato S. Guimaraes, « Sous la casquette de José Luis Guerin », [en ligne] le 26 novembre 2012 [consulté le 25/03/2014]. Disponible sur : http:// www.revuezinzolin.com/2012/12/sous-la-casquette-de-jose-luis-guerin/

MEKAS Jonas, « Le Film-Journal », trad. de l'anglais par Dominique Noguez, dans Françoise Bonnefoy, Danièle Hibon (dir.), Jonas Mekas, catalogue de la rétrospective (Paris, Galerie nationale du Jeu de Paume, 15 décembre 1992 au 31 janvier 1993 ; Marseille, Centre de la vieille charité, Maison méditerranéenne de l'image, 5 au 18 mai 1993), Paris, Éditions du Jeu de Paume, Réunion des musées nationaux, 1992, pp. 47-56. [Le titre original de la conférence est « The Diary Film ». 1 ère éd. P. Adams Sitney (dir.), The Avant-garde film - a reader of theory and criticism, New York, University Press, Anthology Film Archives Series, 1978].

MEKAS Jonas, Lettres de nulle part / Letters from nowhere, trad. du lituanien par Marielle Vitureau en français et Laima Sruoginis en anglais, Paris, Éditions Paris Expérimental, 2003. [1 1 ère éd. Laiškai iš Niekur, Vilnius, Baltos Lankos, 1997].

MEKAS Jonas, Je n'avais nulle part où aller, trad. de l'américain par Jean-Luc Mengus, Paris, P.O.L,

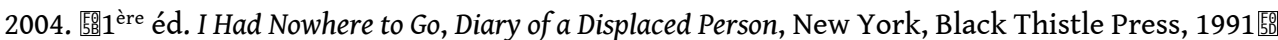

NANCY Jean-Luc, À l'écoute, Paris, Éditions Galilée, 2002.

RAMONEDA Josep, « Retour à l'artisanat », dans Jordi Balló (dir.), Todas las cartas : correspondencias filmicas, catalogue de l'exposition (Mexico du 27 avril au 31 juillet 2011, Barcelone du 12 octobre 2011 au 19 février 2012), Barcelone, Éditions Intermedio, avec la coopération du Centre de culture contemporaine de Barcelone, Acción Cultural Española, le Centre culturel universitaire Tlatelolco, la Casa Encendida de Madrid, 2011, pp. 328-329.

\section{Filmographie}

Lettre 1.

GUERIN José Luis, Carta a Jonas Mekas nº, 8 novembre 2009, 4’53"

Lettre 2 .

MEKAS Jonas, A Letter to José Luis \# 1, janvier 2010, 9'41"

Lettre 3.

GUERIN José Luis, Carta a Jonas Mekas n², mars 2010, 7'22"

Lettre 4 .

MEKAS Jonas, A Letter to José Luis \# 2, avril 2010, 9'04"

Lettre 5.

GUERIN José Luis, Carta a Jonas Mekas n³, mai 2010, 9'39"

Lettre 6. 
MEKAS Jonas, A Letter to José Luis \# 3, juillet 2010, 13'11"

Lettre 7.

GUERIN José Luis, Carta a Jonas Mekas n4, novembre 2010, 9’48”

Lettre 8.

MEKAS Jonas, A Letter to José Luis \# 4, janvier 2011, 19'47"

Lettre 9.

GUERIN José Luis, Carta a Jonas Mekas n5, avril 2011, 14'52"

\section{NOTES}

1. Les "paires" de cinéastes ainsi constituées sont les suivantes : José Luis Guerin-Jonas Mekas, Albert Serra-Lisandro Alonso, Isaki Lacuesta-Naomi Kawase, Jaime Rosales-Wang Bing, Fernando Eimbeke-So Yong Kim.

2. Extrait de la timeline retraçant les différentes étapes du projet et des échanges entre José Luis Guerin et Jonas Mekas, dans Jordi Balló (dir.), Todas las cartas : correspondencias filmicas, catalogue de l'exposition (Mexico du 27 avril au 31 juillet 2011, Barcelone du 12 octobre 2011 au 19 février 2012), Barcelone, Éditions Intermedio, avec la coopération du Centre de culture contemporaine de Barcelone, Acción Cultural Española, le Centre culturel universitaire Tlatelolco, la Casa Encendida de Madrid, 2011, p. 351.

3. Josep Ramoneda, «Retour à l'artisanat », dans Jordi Balló (dir.), Todas las cartas : correspondencias filmicas, ibid., p. 328.

4. Josep Ramoneda, ibid., p. 329.

5. Nous employons cette formulation en référence au «tiers-cinéma », inspiré par Patrick Leboutte, défini comme un cinéma «aux confins du documentaire, de la fiction, de la littérature, des arts plastiques [...] un lieu hybride, insaisissable ", dont le cinéaste Vincent Dieutre rédige l'abécédaire. À l'entrée «lettre », nous pouvons lire : « Le tierscinéma aime à considérer le cinéma comme une écriture. De là lui vient sans doute cette volonté de revisiter les écritures mineures. Jusque-là abîmé dans l'épique et le romanesque, le cinéma peut dorénavant s'écrire en forme de journal intime ou de lettre. [...] Le tiers-cinéma revendique cette prise de parole publique du privé : le cinéma est par essence une révélation. Afin de rester apocalyptique, cette projection du soi hors de soi est la chance du cinéma. Le film devient une lettre à l'adresse ouverte. " Vincent Dieutre, « Abécédaire pour un tiers-cinéma », La lettre du cinéma, n²1, 2003, pp. 75-85.

6. Guy Cornut, La Voix, Paris, Presses Universitaires de France, 2009, p. 43 [1 ère éd. 1983].

7. En 2010, Dos Cartas a Ana (28 minutes) est également une lettre filmée, un dialogue entre cinéma et peinture, prologue à l'installation « La Dama de Corinto ».

8. États-Unis, 1969, 16mm, 173 minutes, noir et blanc et couleur. Tourné entre 1964 et 1968, assemblé en 1968-1969.

9. Jonas Mekas baptise ses films à partir de Walden « films-journaux », lors d'une conférence donnée le 26 août 1972 au Flaherty Seminar (Vermont), à propos de Réminiscences d'un voyage en Lituanie (1972). Jonas Mekas, « Le Film-Journal », trad. de l'anglais par Dominique Noguez, dans Françoise Bonnefoy, Danièle Hibon (dir.), Jonas Mekas, catalogue de la rétrospective (Paris, Galerie nationale du Jeu de Paume, 15 décembre 1992 au 31 janvier 1993 ; Marseille, Centre de la vieille charité, Maison méditerranéenne de l'image, 5 au 18 mai 1993), Paris, Éditions du Jeu de Paume, Réunion 
des musées nationaux, 1992, pp. 47-56 [Le titre original de la conférence est « The Diary

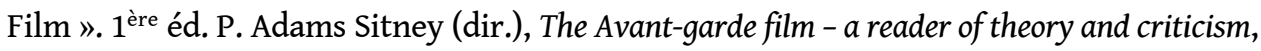
New York, University Press, Anthology Film Archives Series, 1978].

10. États-Unis, 1979, 16mm, 96 minutes, couleur. Tourné 1977, assemblé en 1979.

11. États-Unis, 1992, vidéo, 228 minutes (nouveau montage), couleur.

12. États-Unis, 1997, vidéo, 88 minutes, couleur.

13. États-Unis/Lituanie, 1997, vidéo, 75 minutes, couleur. Ces deux films rappellent le titre de son ouvrage Lettres de nulle part, publié également en 1997, composé de lettres écrites entre 1994 et 1995 pour le Journal du paysan en Lituanie, dans lesquelles Mekas parle de son pays d'origine, de ses habitants, de ses artistes, de la société moderne... Jonas Mekas, Lettres de nulle part / Letters from nowhere, trad. du lituanien par Marielle Vitureau en français et Laima Sruoginis en anglais, Paris, Éditions Paris Expérimental, 2003 [1 ${ }^{\text {ère }}$ éd. Laiškai iš Niekur, Vilnius, Baltos Lankos, 1997].

14. États-Unis, 2004, vidéo, 80 minutes, couleur.

15. Jon Davies, «Brief Glimpses of Beauty: Jonas Mekas at the 2005 Venice Biennale », Cinemascope, $n^{\circ} 25$, [En ligne] 2005 [Consulté le 31/03/2014]. Disponible sur : http:// www.mayastendhalgallery.com/jonas_cinema_scope

/index.html. Article publié à l'occasion de l'exposition de Mekas intitulée « Celebration of the Small and Personal in the Time of Bigness » au Pavillon Lituanien de la $51^{\text {ème Biennale }}$ de Venise, du 12 juin au 6 Novembre 2006. « Mekas has claimed that the letter - an intimate communication between two individuals - has now become his preferred mode in both video and poetry. » [Notre traduction] Nous trouvons aussi des traces de cette pratique dans son journal écrit, à travers les lettres adressées à une Pénélope imaginaire, Mekas s'identifiant au personnage d'Ulysse, dans Jonas Mekas, Je n'avais nulle part où aller, trad. de l'américain par Jean-Luc Mengus, Paris, P.O.L, 2004 㷂1 1 ìre éd. I Had Nowhere to Go, Diary of a Displaced Person, New York, Black Thistle Press, 1991医, pp. 410-411.

16. Maya Deren, «Amateur versus professional », dans Essential Deren, collected writings on film by

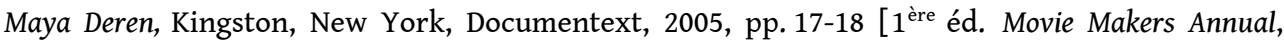
1959].

17. Stan Brakhage, «Défense de l'amateur », trad. de l'américain par Pierre Camus, dans Yann Beauvais, Jean-Michel Bouhours (dir.), Le Je filmé, catalogue de l'exposition (Paris, Musée national d'art moderne, 31 mai au 2 juillet 1995), Paris, Éditions du Centre Pompidou, 1995, pp. 1969-1960 [1 1 ère éd. « In Defense of amateur », dans Filmmakers Newsletters 4 (9-10), été 1971].

18. Stan Brakhage, « Défense de l'amateur », op.cit., p. 1969.

19. José Luis Guerin, septembre 2012, trad. de l'espagnol par André Gabastou. Texte introductif à la brochure accompagnant sa rétrospective au Centre Pompidou, à Paris, du 30 novembre 2012 au 7 janvier 2013, dans le cadre de la manifestation « José Luis Guerin / Jonas Mekas, cinéastes en correspondance », p. 2.

20. Cette formulation n'est pas traduite en français, les sous-titres conservent la phrase originale en anglais. Mais nous pourrions la traduire par « je réagis à la vie».

21. "Quand on tourne avec une Bolex, on la tient à un endroit, pas exactement à l'emplacement du cerveau, un petit peu plus bas, et pas exactement à l'endroit du cœur, légèrement plus haut... Et alors on remonte le ressort, on lui donne une vie artificielle...[... ] », dans Jonas Mekas, « Le Film-Journal », op.cit., p. 53.

22. Nous pouvons également penser à Alain Cavalier, qui fait corps avec son outil et parle de l'intégration de la caméra par son corps en ces termes : «Avec la caméra à votre œil et le son dans vos oreilles, c'est votre corps qui fait le film. Quelquefois on entend les pulsations de mon cœur.» dans Alain Cavalier, entretien avec Jean-Pierre Limosin et Serge Toubiana, «Les pulsations de mon cœur, le 29 avril 1996 », Les Cahiers du Cinéma, n504, juillet/août 1996, p. 43. 
23. Nous voyons seulement son ombre ou son reflet, étrangement déformés par une petite caméra surmontée d'un micro, témoignant d'un rapport au monde visuel et sonore.

24. Jean-Louis Comolli, "L'oral et l'oracle, séparation du corps et de la voix», Images Documentaires, $\mathrm{n}^{\circ} 55 / 56,1^{\mathrm{er}}$ trimestre 2006, Paris, p. 26.

25. José Luis Guerin, propos recueillis par Renato S. Guimaraes, « Sous la casquette de José Luis Guerin », [en ligne] le 26 novembre 2012 [consulté le 25/03/2014]. Disponible sur : http://www.revuezinzolin.com/2012/12/sous-la-casquette-de-jose-luis-guerin/

26. Alain Bergala, "Je t'écris ces images...», dans Jordi Balló (dir.), Todas las cartas : correspondencias filmicas, op.cit. p. 333.

27. «Paris, 8 novembre 2009. Cher Jonas [...] ». [Nous traduisons]

28. "Cher José Luis, merci pour ta lettre, voici à présent ma courte lettre. » Nous pouvons noter que « Dear José Luis » apparaît d'abord à l'écrit, par le biais d'un intertitre rédigé à la main, avant d'être répété à l'oral.

29. «Mon ami en cinéma ».

30. Ce qui est très différent de la correspondance filmée échangée entre Albert Serra et Lisandro Alonso par exemple. Au film de 23 minutes envoyé par Alonso à Serra, ce dernier répond par un film d'une durée de 146 minutes, créant un déséquilibre radical dans la correspondance. Celle-ci ne se poursuivra pas au-delà de ces deux échanges. Sin título (Carta para Serra), de Lisandro Alonso, Argentine/Espagne, 2011, vidéo, 23 minutes, couleur. El Senyor ha fet en mi meravelles, d'Albert Serra, Espagne, 2011, vidéo, 146 minutes, couleur.

31. Alain Bergala, "Je t'écris ces images...», dans Jordi Balló (dir.), Todas las cartas : correspondencias filmicas, op.cit. p. 338.

32. Nous entendons pas l'emploi du terme « variations » la production de phrases musicales qui diffèrent sensiblement les unes des autres, mais qui se rapportent à un même thème, qui serait dans le cas de notre étude, l'échange épistolaire cinématographique.

33. Guerin se rend au lac de Walden, où Henry David Thoreau vécut pendant deux ans et écrivit Walden (ou la vie dans les bois), publié en 1854. Livre fondateur pour Mekas, il intitule son premier «film-journal " composé à partir de ses notes filmées et sonores, Diaries, Notes and Sketches (Also known as Walden).

34. «Cher Jonas".

35. Alain Fleischer, L'Accent : une langue fantôme, Paris, Éditions du Seuil, 2005, p. 92.

36. Ibid., p. 78.

37. C'est-à-dire « invité ». Guerin réalise un film du même nom en 2010 (Espagne, 2010, vidéo, 127 minutes, noir et blanc). Cette position d'invité est finalement déjà contenue dès les premières minutes du film, par le biais des images extraites du film Funny Face (Drôle de frimousse) (1957) de Stanley Donen. Le personnage interprété par Audrey Hepburn, Joe Stockton, profite d'une invitation à Paris à la demande du photographe Dick Avery (Fred Astaire), pour détourner l'objet de son voyage.

38. Jordi Balló, « Les Lettres filmées », dans Jordi Balló (dir.), Todas las cartas : correspondencias filmicas, op.cit., p. 330.

39. Jean-Luc Nancy, À l'écoute, Paris, Éditions Galilée, 2002, p. 17.

40. Guerin semble nous donner un élément de réponse dans sa première lettre, avec les images extraites de Funny Face.

41. Jonas Mekas dit, durant cette séquence : « C'est notre histoire, notre passé, sanglant, plein de sang. "

42. Alain Bergala, «Je t'écris ces images...», op.cit., p. 337.

43. Ce film, réalisé depuis, est Out-Takes from the Life of a Happy Man, États-Unis /

Angleterre, 2012, 16mm, 68 minutes, couleur, sur une commande de la Serpentine Gallery. 
44. En réponse aux voyages de Mekas en Pologne et en Slovaquie sur des lieux de souffrance, Guerin évoque sa visite du camp d'Auschwitz en Pologne.

45. Daniel Deshays, Entendre le cinéma, Paris, Éditions Klincksieck, 2010, p. 99.

46. Guerin parle de la différence pour lui entre "filming» et "taping», et reprend également l'expression "filming mistakes », employée par Mekas à propos des événements traumatiques de l'Histoire dont il a enregistré les traces.

47. Il évoque alors la situation de Jonas Mekas, fils de paysan lituanien exilé aux États-Unis. Guerin, qui a choisi d'être un "invité errant », s'imagine comme étant " une espèce de copie plus à l'aise » de ces personnes déplacées.

48. « le cinéaste en personne".

49. Guerin dit : "Yo pensaba hacerlo de un modo domestico, casi intimo ", c'est-à-dire "J'avais prévu de le faire sur un mode familier, presque intime ».

50. Nous remarquons que Guerin, plutôt que de s'attarder sur les tombes, filme ce qui est vivant, que ce soit les femmes qu'il rencontre ou la nature luxuriante autour. Mekas, lors de sa visite du cimetière profané en Pologne, filmait également les vivants ainsi que la végétation abondante.

51. Nous retrouvons dans les bandes sonores de l'œuvre cinématographique de Jonas Mekas (en particulier ses films argentiques) des éléments inspirés par John Cage, lui-même adepte du bouddhisme zen.

\section{RÉSUMÉS}

Entre novembre 2009 et avril 2011, les cinéastes Jonas Mekas et José Luis Guerin entretiennent une correspondance filmée. Si cette pratique est encore inédite pour Guerin, Mekas a quant à lui régulièrement adressé ses films à d'autres. Dans les neuf lettres qu'ils échangent, la voix est ce qui permet le passage d'une forme traditionnellement écrite à un objet cinématographique. Les cinéastes parfois visibles, parfois hors champ ont la particularité de porter la caméra, d'être à la fois à l'image et au son. Leurs corps sont les lieux d'émission de la voix, mais aussi de l'écoute, couple indissociable au sein de l'espace cinématographique singulier que crée la lettre filmée. La voix est transmission de soi, mais aussi écho de l'autre, voûte invisible qui permet d'adresser des images et des sons.

Between November 2009 and April 2011, filmmakers Jonas Mekas and José Luis Guerin have corresponded by means of filmed letters. While this practice was still new for Guerin, Mekas had already regularly sent his films to others. Through their nine letters, their voices allow the transition from a traditional literary form to a filmic object. Whether they are visible or off screen, the filmmakers both hold the camera, recording both sound and image by themselves. Their bodies are at the same time the sources of the voice and of listening. Both are inseparable in the singular filmic space created by the filmed letters. The voice is a transmission of the self, it is also an echo of the addressee who acts as an invisible keystone in the sending of images and sounds.

INDEX

Mots-clés : Autobiographie, lettre filmée, voix, regard, écoute, Jonas Mekas, José Luis Guerin 


\section{AUTEUR}

\section{CÉCILE TOURNEUR}

Docteur en Esthétique, Sciences et Technologies du Cinéma et de l'Audiovisuel (Université Paris 8). Sa thèse portait sur la qualification de la voix dans les films de Jonas Mekas entre 1966 et 2009. Elle enseigne par ailleurs l'histoire du cinéma indépendant américain des années 1960 et a notamment collaboré au Dictionnaire de la pensée du cinéma dirigé par Antoine de Baecque et Philippe Chevalier en 2012. 